\title{
EDITORIAL
}

\section{When and how to manage enteral feeding intolerance?}

\author{
Yaseen M. Arabi ${ }^{1 *} \mathbb{D}$, Annika Reintam Blaser ${ }^{2,3}$ and Jean-Charles Preiser ${ }^{4}$
}

(c) 2019 Springer-Verlag GmbH Germany, part of Springer Nature

\section{Introduction}

In this issue of Intensive Care Medicine, Heyland et al. reported the results of the PROMOTE Trial [1]. In this international multicenter (20 sites, 4 countries) randomized double-blind study, 120 critically ill patients who had enteral feeding intolerance (EFI) were randomized to receive ulimorelin or metoclopramide for 5 days. A volume-based feeding protocol was employed, with a starting feeding rate of $40 \mathrm{~mL} / \mathrm{h}$ and maximum rate of $150 \mathrm{~mL} / \mathrm{h}$. Gastric residual volumes (GRV) were measured every $6 \mathrm{~h}$, and the feeding rate was increased if GRV was $<500 \mathrm{~mL}$, and reduced if GRV was $\geq 500 \mathrm{~mL}$; if $\mathrm{GRV} \geq 500 \mathrm{~mL}$ persisted in two consecutive measurements with feeding $10 \mathrm{~mL} / \mathrm{h}$ beyond day 1 , the study drug was discontinued.

The study found no difference between ulimorelin and metoclopramide in the primary endpoint of percentage of daily protein prescription (DPP) over 5 days of treatment. There were no differences in the secondary endpoints of feeding success, gastric emptying assessed by paracetamol absorption, incidence of recurrent EFI, vomiting or regurgitation, aspiration, and pulmonary infection. The study highlighted some uncertainties and the need for harmonization.

\section{Definition of enteral feeding intolerance}

EFI is common in the critically ill, and often results in not achieving nutritional targets. It may also be associated with significant morbidity, leading to increased mortality and ICU length of stay [2].

\footnotetext{
*Correspondence: arabi@ngha.med.sa

${ }^{1}$ College of Medicine, King Saud bin Abdulaziz University for Health Sciences (KSAU-HS) and King Abdullah International Medical Research Center (KAIMRC), P.O. Box 22490, Riyadh 11426, Kingdom of Saudi Arabia Full author information is available at the end of the article
}

However, there is considerable variation in defining what constitutes EFI. A systematic review of 72 studies estimated prevalence of EFI of $38 \%$ (95\% CI 31-46), but demonstrated large variability in defining EFI [2]. Upper gastrointestinal tract intolerance reflected by large GRVs (with or without other gastrointestinal symptoms) was used in $63 / 72$ studies, with a median volume defining a "large" GRV of $250 \mathrm{~mL}$ (range 75-500 mL) [2].

\section{Efficacy and safety of prokinetic agents}

Prokinetic agents to improve gastric emptying are used off-label in critically ill patients. A systematic review of 13 randomized controlled trials $(n=1341$ critically ill patients) assigned to receive a prokinetic agent (metoclopramide, erythromycin, domperidone) or placebo confirms the reduction in GRVs (RR 0.69; 95\% CI 0.52-0.91) and EFI (17.3\%; 95\% CI 5-26.8\%) by prokinetics, with no difference in vomiting, diarrhea, pneumonia or mortality [3]. Importantly, only $5 / 13$ studies reported EFI as an outcome, while increased GRV, vomiting and diarrhea were commonly reported [3].

Side effects and tachyphylaxis are of concern in prokinetic use. In addition, the PROMOTE study demonstrated some differences in the safety profile of the two drugs: serious adverse events occurred more often in the ulimorelin group, but adverse events leading to study drug discontinuation, including atrial fibrillation, were more common in the metoclopramide group. Although most of these differences were not statistically significant, the sample size was too small to draw definitive conclusions.

\section{Indications and contraindications for prokinetic therapy}

There is a lack of consensus on indications and contraindications for prokinetic therapy in critically ill patients. EFI can be considered as an adaptive

\section{6 Springer}


mechanism during the early course of critical illness [4]. The decrease in plasma ghrelin levels reported by two independent teams was used to support the use of ulimorelin, which acts as a ghrelin agonist $[5,6]$. However, the decline in ghrelin release could be considered a signaling pathway sent by the upper gastrointestinal tract as a component of the adaptive response leading to anorexia during the early course of critical illness. Additionally, uncertainty around diagnostic criteria for gastrointestinal dysmotility distal to the stomach warrants caution towards treatment with gastroprokinetics. Improving gastric emptying in cases where the problem lies caudally may worsen bowel distension, leading to complications. Therefore, it is unclear how aggressive we should be in attempting to attain nutritional targets in the first few days of critical illness in patients with EFI. Administration of full calories (100\% of energy expenditure) in the early acute phase has not been shown to be beneficial and is not currently recommended [7-11]. The NUTRIREA-2 trial suggests that attempting to achieve full-dose enteral feeding rapidly in patients at risk for gastrointestinal injury may be associated with adverse events [12]. The optimal dose of protein in the acute phase of critical illness is even less certain, and we await publication of data from randomized controlled trials $[13,14]$.

Accordingly, one approach to patients with one episode of large GRV is to transiently reduce the nutritional target, and consider prokinetic agent only if large GRVs persist or are associated with other gastrointestinal symptoms, whereas small bowel distension is excluded [15]. Additionally, duration of treatment needs to be limited to achieve optimal benefit: risk ratio.

Volume-based protocol was used together with prokinetic agents in all patients in PROMOTE Trial; yet only $51.6 \%$ and $55.2 \%$ of ulimorelin and metoclopramide patients in the intention-to-treat population achieved feeding success over the 5 days of treatment $(\geq 80 \%$ of daily protein prescription). At the same time, episodes of EFI declined progressively over the 5 study days in both groups. While all patients had EFI prior to randomization, only $25 \%$ had EFI on day 1 , and only $15 \%$ of patients continued to experience EFI episodes by day 5; only $50.0 \%$ had EFI recurrence during the trial.

These findings highlight the transient nature of upper gastrointestinal tract EFI in many ICU patients and raise several important questions. Should a single episode of large GRV trigger prokinetic treatment? In addition, could the treatment effect of prokinetic agents have been "diluted" by including patients with transient EFI? What is the optimal duration of therapy in responders versus nonresponders?
In summary, ulimorelin and metoclopramide administration demonstrated comparable efficacy in treatment of EFI defined as a single GRV $\geq 500 \mathrm{ml}$. In a considerable proportion of patients, the feeding target was not achieved despite volume-based feeding, prokinetic administration for 5 days and a relatively low number of patients with recurrent EFI as defined based on large GRV.

Research addressing EFI and its treatment needs to continue. In the search for new effective and safe molecules, an ultimate goal should be kept in mind: identification of indications for prokinetic treatment (along with a clear definition of EFI) to achieve the optimal benefitto-risk ratio for such treatment.

\section{Author details}

${ }^{1}$ College of Medicine, King Saud bin Abdulaziz University for Health Sciences (KSAU-HS) and King Abdullah International Medical Research Center (KAIMRC), P.O. Box 22490, Riyadh 11426, Kingdom of Saudi Arabia. ${ }^{2}$ Department

of Intensive Care Medicine, Lucerne Cantonal Hospital, Lucerne, Switzerland.

${ }^{3}$ Department of Anaesthesiology and Intensive Care, University of Tartu, Tartu, Estonia. ${ }^{4}$ Department of Intensive Care, Erasme University Hospital, Université Libre de Bruxelles, Brussels, Belgium.

\section{Conflict of interest}

Dr. Yaseen Arabi institution received funding from King Abdullah International Medical Research Center, Riyadh, Saudi Arabia, and King Abdulaziz City for Science and Technology, Riyadh, Saudi Arabia, for the studies on permissive underfeeding and for biomarker substudies. Dr Annika Reintam Blaser received speaker fees from Fresenius and Nestlé and a study grant from Fresenius given to University of Tartu. Dr Jean-Charles Preiser received speaker fees from Baxter, Fresenius, Nestle and Nutricia.

\section{Publisher's Note}

Springer Nature remains neutral with regard to jurisdictional claims in published maps and institutional affiliations.

Received: 29 April 2019 Accepted: 30 April 2019

Published online: 24 May 2019

\section{References}

1. Heyland DK et al (2019) A multicenter, randomized, double-blind study of ulimorelin and metoclopramide in the treatment of critically ill patients with enteral feeding intolerance: PROMOTE trial. Intensive Care Med 45(5):647-656

2. Blaser AR, Starkopf J, Kirsimagi U, Deane AM (2014) Definition, prevalence, and outcome of feeding intolerance in intensive care: a systematic review and meta-analysis. Acta Anaesthesiol Scand 58(8):914-922

3. Lewis K, Alqahtani Z, Mclntyre L et al (2016) The efficacy and safety of prokinetic agents in critically ill patients receiving enteral nutrition: a systematic review and meta-analysis of randomized trials. Crit Care 20(1):259

4. Hill NE, Murphy KG, Singer M (2012) Ghrelin, appetite and critical illness. Curr Opin Crit Care. 18(2):199-205

5. Nematy M, O'Flynn JE, Wandrag L et al (2006) Changes in appetite related gut hormones in intensive care unit patients: a pilot cohort study. Crit Care 10(1):R10

6. Santacruz CA, Quintairos A, Righy C et al (2017) Is there a role for enterohormones in the gastroparesis of critically ill patients? Crit Care Med 45(10):1696-1701 
7. Arabi YM, Aldawood AS, Haddad SH et al (2015) Permissive underfeeding or standard enteral feeding in critically ill adults. N Engl J Med 372(25):2398-2408

8. Rice TW, Mogan S, Hays MA, Bernard GR, Jensen GL, Wheeler AP (2011) Randomized trial of initial trophic versus full-energy enteral nutrition in mechanically ventilated patients with acute respiratory failure. Crit Care Med 39(5):967-974

9. National Heart L, Blood Institute Acute Respiratory Distress S, Rice TW et al (2012) Initial trophic vs full enteral feeding in patients with acute lung injury: the EDEN randomized trial. JAMA J Am Med Assoc 307(8):795-803

10. Target Investigators ftACTG, Chapman M, Peake SL et al (2018) Energydense versus routine enteral nutrition in the critically ill. N Engl I Med 379(19):1823-1834
11. Singer $P$, Blaser AR, Berger MM et al (2019) ESPEN guideline on clinical nutrition in the intensive care unit. Clin Nutr 38(1):48-79

12. Reignier J, Boisrame-Helms J, Brisard L et al (2018) Enteral versus parenteral early nutrition in ventilated adults with shock: a randomised, controlled, multicentre, open-label, parallel-group study (NUTRIREA-2). Lancet 391(10116):133-143

13. Arabi YM, Casaer MP, Chapman M et al (2017) The intensive care medicine research agenda in nutrition and metabolism. Intensive Care Med 43:1239-1256

14. Preiser JC (2018) High protein intake during the early phase of critical illness: yes or no? Crit Care 22(1):261

15. Koekkoek K, van Zanten ARH (2018) Nutrition in the ICU: new trends versus old-fashioned standard enteral feeding? Curr Opin Anaesthesiol 31(2):136-143 